\title{
Review
}

Journal of Innate

Immunity
J Innate Immun 2009;1:123-135

DOI: 10.1159/000163037
Received: May 30, 2008

Accepted after revision: July 29, 2008

Published online: October 8, 2008

\section{Role of Intestinal Mucins in Innate Host Defense Mechanisms against Pathogens}

\author{
Poonam Dharmani Vikas Srivastava Vanessa Kissoon-Singh Kris Chadee \\ Gastrointestinal Research Group, Faculty of Medicine, University of Calgary, Calgary, Alta., Canada
}

\section{Key Words}

Mucin $\cdot$ Innate immunity $\cdot$ Inflammatory bowel disease $\cdot$

Toll-like receptors

\begin{abstract}
Gastrointestinal mucins produced by goblet cells comprise the main structural components of the mucus layer. Mucins play a critical role in the maintenance of mucosal homeostasis and are responsible for the differential effector and regulatory responses against a plethora of microorganisms, including commensals and pathogens. In this review, we present a comprehensive overview on mucin biology, its properties, classification and gene assembly. We also consider the structure of the mucin gene, its proteins and its role in innate host defenses. We compare the various mucin secretagogues and the differential regulatory pathways involved in mucin biosynthesis and secretion during normal and diverse pathogenic conditions. Finally, we summarize the putative uncharted aspects of mucin-derived innate host defenses, whose exploration will help drug developers to identify factors that can strengthen mucosal integrity and will facilitate basic science research into curative treatments for gastrointestinal diseases.

Copyright $\odot 2008$ S. Karger AG, Basel
\end{abstract}

\section{Introduction}

The mucosal surface of the gastrointestinal tract is a complex organization of epithelium, immune cells and resident microbiota [1]. The intestinal epithelium is covered by specialized mucin-producing goblet cells, which together with other substances such as antimicrobial peptides, lysozymes and resident microbiota collectively constitute 'innate immunity' and form the front line of defense against pathogenic microorganisms [2]. The luminal surface, which is made up of epithelial cells and mucins, acts as a protective barrier that separates the contents of the harsh luminal environment from the layers of tissue comprising the internal milieu.

Goblet cells, found in the columnar epithelium, secrete high molecular weight glycoproteins called mucins. These mucins have a high negative surface charge and a large hydration capacity. Mucins act as the main structural component of the mucus layer, giving rise to its polymeric, viscoelastic and protective properties. Mucins are mainly found at the periphery of epithelial cells and their extracellular environment or covering epithelial cells. The surface of the gastrointestinal epithelium is continually exposed to numerous macromolecules and microorganisms including chemical irritants, digested foods, toxins, resident bacteria, intestinal pathogens and their prod- 
ucts [3]. The mesh-like structure of the mucin gel not only impedes the diffusion of offending macromolecules but also serves numerous other functions. These include lubrication for the passage of particulate matter, maintenance of a hydrated layer over the epithelium, forming a barrier to noxious substances and creating a permeable gel layer for the exchange of gases and nutrients with the underlying epithelium [4]. The best-established function is the prevention of the entry of enteric pathogens. The mucus layer contains binding sites for resident microbiota and maintains high concentrations of secretory IgA to prevent attachment and binding of pathogenic microorganisms [5]. In this review, we present an update on the biology of mucins, their properties and expression patterns under normal and pathogenic conditions. Our aim is to present a cumulative overview of the diverse range of functions performed by these highly specialized protein moieties in general and, in particular, their role as an innate immune defense barrier.

\section{Classification and Expression Profile of Mucins}

To date, 21 different mucin genes have been identified, cloned and partially sequenced in humans $[6,7]$, and the majority of their homologues have been identified in mice and rats [8]. Mucins are subdivided into secretory and membrane-bound forms, depending on their structure and location. Secreted mucins contribute to the formation of the mucus gel but the function of membranebound mucins is not well characterized, despite the fact that they are located on the surface of epithelial cells throughout the body. A summary of the mucins, their tissue distribution and expression is listed in table 1. MUC2 is the major mucin, produced by the goblet cells in the intestinal epithelium [9] and will be the focus of this review.

\section{Mucin Structure}

Mucins are large glycoproteins that range in size from $0.5 \times 10^{6}$ to $25 \times 10^{6} \mathrm{Da}$ [10]. The mucin monomer accounts for approximately $1.5 \times 10^{6} \mathrm{Da}$, but this may be an underestimate due to allelic variations in the variable number of tandem repeats [11]. The protein core of a typical mucin molecule contains mucin domains that consist of tandem repeats rich in threonine, proline and/or serine, the hydroxyl residues of which are heavily substituted with $O$-linked oligosaccharides [12]. The carbohydrate content of a secretory mucin can account for up to $80 \%$ of its dry weight and gives it a unique 'bottle-brush' appearance. The densely packed oligosaccharides serve to protect the protein core from proteases, thereby preserving the integrity of the mucin polymer [10]. The addition of sulfate and $O$-acetyl-substituted sialic acid to terminal mucin oligosaccharides confers additional resistance to degradation by glycosidases [13]. The process of glycosylation confers many of the general properties of mucins including high-charge density from sialic acid and sulfate residues, protease resistance and water holding capacity (hydration) [3]. Evidence suggests that glycosylation can be altered in response to mucosal infection and inflammation, and this may be an important mechanism for mucosal pathogens. The extensive $O$-glycosylation of the mucins protects them from proteolytic enzymes [14].

The $\mathrm{N}$ - and $\mathrm{C}$-terminal regions of mucin are poorly glycosylated compared to the mucin domains and they contain a wide range of amino acids, most notably cysteine residues [10]. Secretory mucins often contain $3 \mathrm{~N}$-terminal von Willebrand factor-like domains (termed D1, D2 and D3), while the C-terminal region contains only one D4 domain. N- and C-terminal cysteine-rich domains are less glycosylated, while the protein core, which is exposed, is resistant to proteolytic enzymes due to a high number of intramolecular disulfide bonds that shields potential cleavage sites from enzymes [15].

\section{Secretion of Mucin}

The secretion of mucins is a multifaceted phenomenon, regulated by a number of different factors. We have categorized this complex process into 2 broad subsections: (1) constitutive or baseline, which is an unregulated low-level secretion, and (2) stimulated or accelerated secretion in response to external stimuli.

\section{Constitutive or Baseline Secretion}

Under normal physiological conditions, goblet cells continually synthesize and secrete mucins to replenish the mucus blanket covering the epithelium. This continual secretion of mucin is necessary to maintain the thickness of the mucus gel, which is constantly being exposed to acids and irritants and is often sloughed off due to peristaltic movements [16].

Mucosal explants of human and rat colons have been shown to continually incorporate radio-labeled mucin 
Table 1. Characteristics of different mucin genes

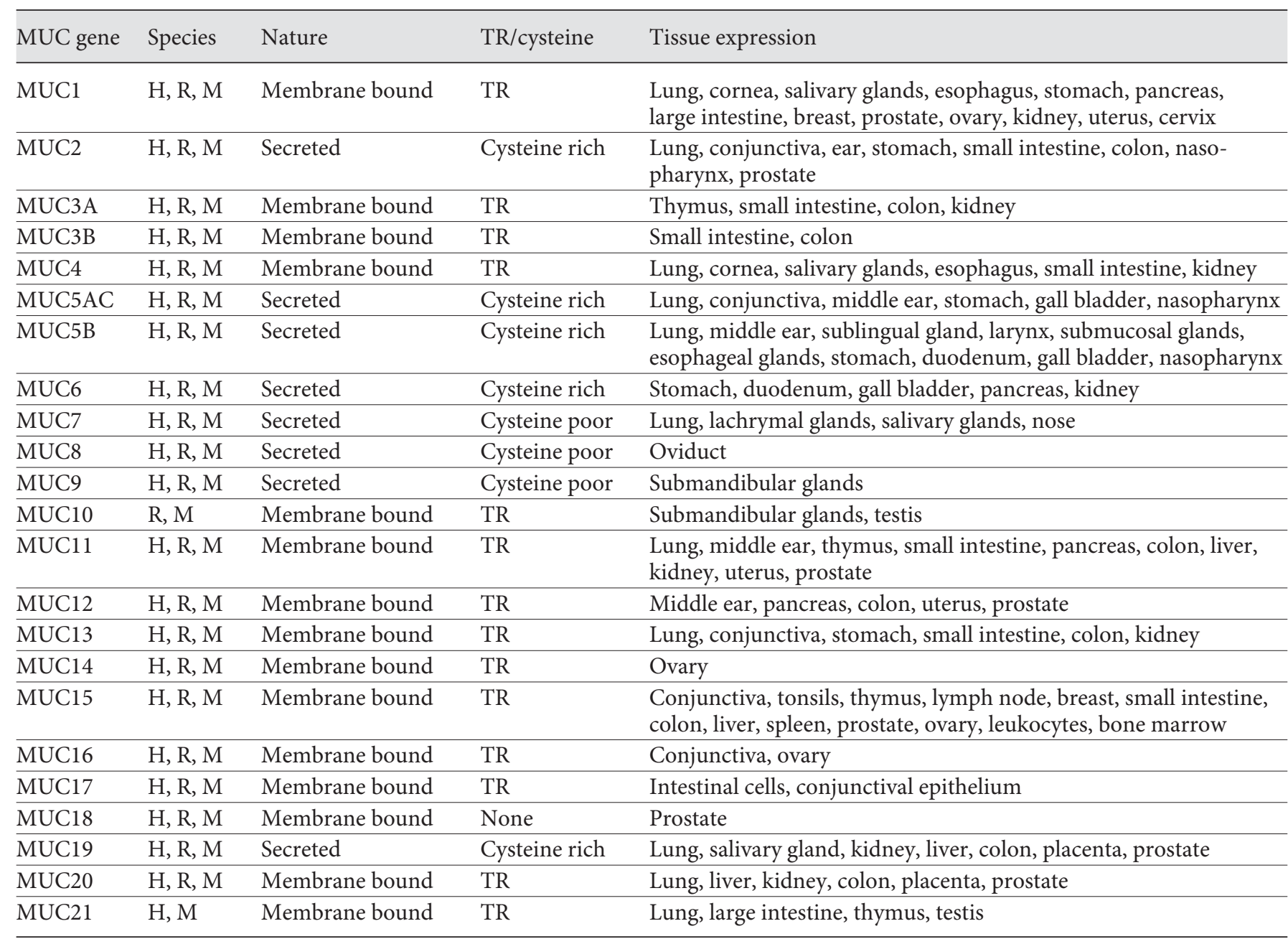

$\mathrm{H}=$ Human; $\mathrm{M}=$ mouse; $\mathrm{R}=$ rat; $\mathrm{TR}=$ tandem repeat.

precursors into mucins [17]. Following synthesis, labeled mucins are then packaged into granules, transported to the cell surface and secreted into the lumen. Little is known about the mechanism of unregulated secretion, but it is not a receptor-mediated event [18]. Rather, it appears to depend on the continuous transport of granules from the Golgi vesicles to the cell surface, which in turn is a microtubule-dependent event [19].

\section{Stimulated or Accelerated Secretion}

When goblet cells are exposed to potent secretagogues, mucin is released in an accelerated fashion [20], either by release of centrally stored mucin granules or by release of single mucin granules one at a time. Release of centrally stored mucin granules is then followed by compound exocytosis of granules and the release of peripheral granules resulting in the cavitations of the deep apical membrane. Mucus secretagogues induce a rapid increase in the number of granules released from the goblet cell, with a maximum rate of exocytosis established 1-3 min after stimulation, followed by a much slower release of mucin granules [21]. A wide array of bioactive factors, including hormones (neuropeptides) and inflammatory mediators (cytokines and lipids), can induce compound exocytosis $[4,22,23]$. Some of the major mucin secretagogues and their modes of action are shown in figure 1.

Stimulated or accelerated mucin secretion is linked to a receptor-mediated event. We have shown that prosta- 
Fig. 1. Modulation of MUC2 expression by inflammatory mediators and mucin secretagogues. Pro- and anti-inflammatory cytokines differentially activate the SAPK/ JNK or the JAK/STAT pathways while mucin secretagogues cause cAMP-mediated activation of CREB. $\mathrm{RA}=$ retinoic acid; $\mathrm{RAR}=$ retinoic acid receptor; $\mathrm{AC}=$ adenylate cyclase; $\mathrm{PKA}=$ protein kinase $\mathrm{A}$; $\mathrm{CREB}=$ cAMP-responsive element; SAPK/ $\mathrm{JNK}=$ stress-activated protein kinase/ c-Jun NH2-terminal kinase; JAK = janus kinase; STAT = signal transducers and activators of transcription.

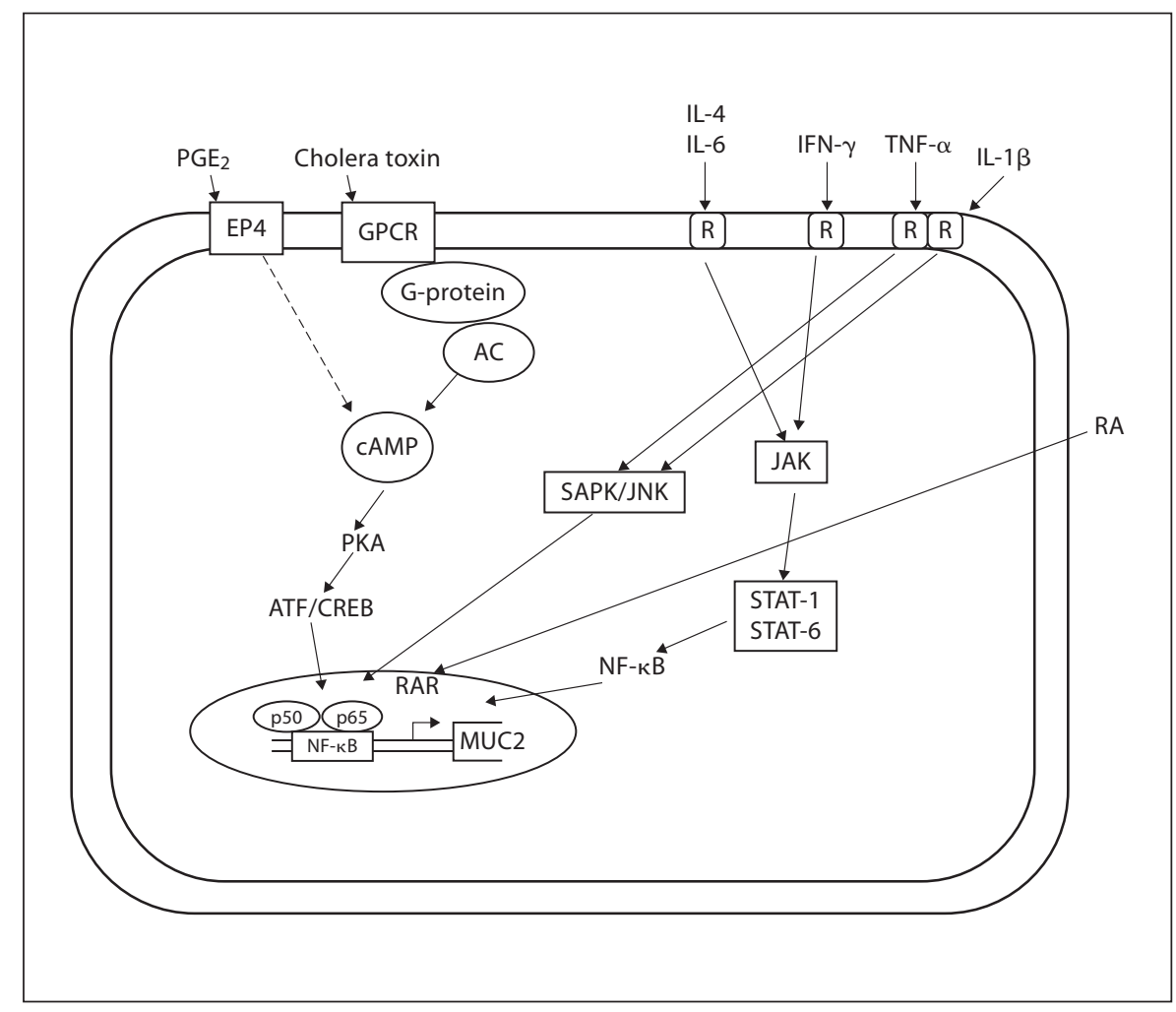

glandin $\mathrm{E}_{2}$ binds the EP4 receptor to stimulate cAMP-dependent exocytosis in LS 174T cells [24]. Wright et al. [25] have shown that prostaglandin $\mathrm{D}_{2}$ evokes mucin secretion in these cells via the DP receptor. Mucin secretagogues are known to signal through various secondary messengers that include intracellular $\mathrm{Ca}^{2+}, \mathrm{cAMP}$ and diacylglycerol, which activates protein kinase $C[26,27]$ to stimulate mucin secretion.

\section{Mucin and Innate Host Defense}

An important aspect of mucosal immunity is the differentiation between self and non-self. Under normal conditions, endogenous bacterial species (aerobic, facultative and anaerobic bacteria) of the microbiota normally localize in the mucus. These all share 'self' signature molecules, or pathogen-associated molecular patterns. These patterns are in turn recognized by Toll-like receptors or other pattern recognition receptors. The host system is highly adapted to commensal organisms through immune adaptation, immune ignorance or mucosal homeostasis [28].

\section{Role of Intestinal Microbes in Mucin Regulation}

The human intestine is colonized by a complex, dynamic microbial ecosystem. A single region of the intestine may contain more than 400 bacterial species, including resident microbiota and a variable array of transient species that temporarily occupy an empty niche [29]. Usually, commensal organisms peacefully coexist with host cells. However, this seemingly ideal balance is sometimes disturbed, which can facilitate the development of inflammatory bowel diseases (IBD). Intestinal microbes may directly affect goblet cell function through the local release of bioactive factors generated by activated epithelial cells or underlying lamina propria cells. Gut microbes can also regulate mucin production by activating different signaling cascades and secretory elements. A representative MUC2 gene with its identified cis-acting elements and their binding sites is shown in figure 2. In the following section, we discuss how mucus secretion, synthesis and chemical composition are altered by microbederived factors. 


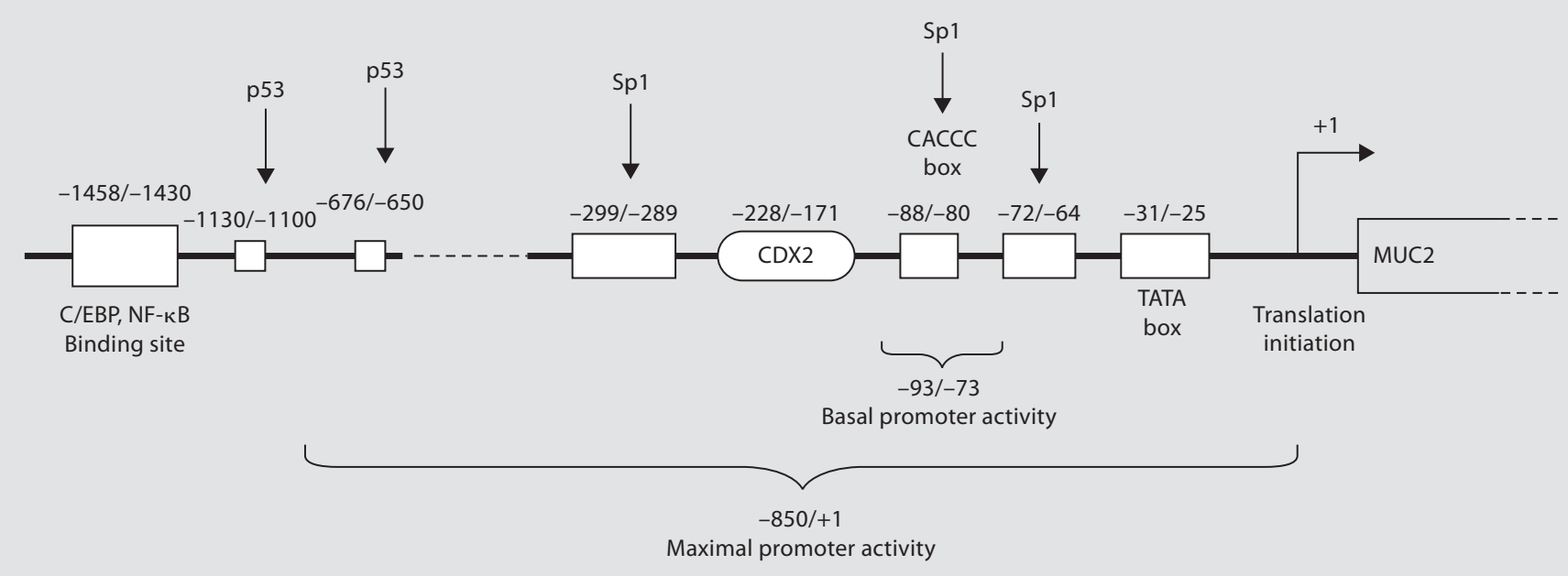

Fig. 2. General organization of a MUC2 promoter and the location of identified cis-acting elements. NF- $\kappa \mathrm{B}$ is the final effector molecule in multiple pathways involved in mucin regulation. Sp1, p53 and other transcription factors bind at various GC-rich boxes in the promoter region. Location is numerically denoted relative to the AUG (+1) translation codon. C/EBP = CCAAT/enhancer binding protein; NF- $\kappa \mathrm{B}=$ nuclear factor $\kappa \mathrm{B} ; \mathrm{CDX} 2=$ caudal-type homeobox transcription factor-2.

\section{Microbe-Derived Factors Alter Mucin Synthesis and Secretion}

The continuity and stability of the mucus layer are critical to intestinal homeostasis, since it forms the primary barrier against pathogenic infections. Interaction with the mucus layer determines the fate of pathogenic infections and the diseases they cause. Mucus offers numerous ecological advantages to intestinal bacteria. For example, mucin represents a direct source of carbohydrates and peptides, as well as exogenous nutrients, including vitamins and minerals. Bacteria capable of colonizing mucus can avoid rapid expulsion via peristalsis of the intestine. In addition, this colonization imparts a growth advantage on these organisms and permits them to exploit underlying signaling pathways. Thus, it is not surprising that mucus secretion is typically enhanced in response to intestinal microbes. Both commensal and pathogenic organisms derive significant benefits from their ability to chemically regulate mucin synthesis or secretion of host goblet cells. Here, we will describe the various pathways that intestinal pathogens use to induce or reduce mucin secretion.
Entamoeba histolytica as a Model for Interactions with Mucin

The protozoan parasite Entamoeba histolytica is one of the most successful pathogens of the gastrointestinal tract. Its success lies in its ability to modulate the mucus barrier to its advantage in several different ways. E. histolytica has a highly specific adhesin for binding to mucin and to host cells [30]. It also has the ability to colonize and live in the mucus layer without causing disease. In some cases, however, the amoeba is able to breach epithelial barrier defenses, which causes amoebic dysentery with severe ulceration of the colonic mucosa [30]. Along with potent mucin secretagogue activity, E. histolytica has the ability to degrade colonic mucins at the site of contact. In a gerbil model of amoebic colitis, we have shown that the luminal mucus barrier and goblet cell mucin stores are depleted prior to amoeba contact with and invasion of the underlying mucosa [27]. While the cause of the mucin depletion is unknown, it is speculated that parasite-derived secretagogues and mucinase activity may deplete mature mucin stores as a means of evading epithelial barrier functions [24]. This host-parasite interaction with the mucus barrier is the best-characterized model to date to discern the distinct events involved in the pathogenesis of colonic disease. 
The parasite's interaction with mucin determines the final fate of amoebic invasion. The most important factor regulating this is the galactose $/ \mathrm{N}$-acetylgalactosamine (Gal/GalNAc) adherence lectin (Gal-lectin) which recognizes and binds with high-affinity galactose and $\mathrm{N}$-acetylgalactosamine residues on colonic mucins and surface receptors of host cells [31]. Gal-lectin binding to target cells is an absolute prerequisite for contact-dependent killing. Thus, blockade of Gal-lectin activity with Gal and/or GalNAc-containing oligosaccharides prevents contact-dependent cytotoxicity [32]. We have shown that Gal and GalNAc sugar residues of purified colonic mucins competitively inhibit amoeba binding to host epithelium by creating a physical barrier between the parasite and the epithelium. Colonic mucin forms a barrier that protects the host and inhibits amoebic adherence to the underlying epithelial cells. We have developed an in vitro model to study the interaction between E. histolytica and colonic mucins [33]. We found that both crude and purified colonic mucins derived from LS $174 \mathrm{~T}$ cells prevented amoebic adherence to Chinese hamster ovary cells. The mucin-secreting LS $174 \mathrm{~T}$ cells, which had a protective mucus barrier, were more resistant to amoeba attack than the mucin-deficient Chinese hamster ovary cell monolayer. Treatment of LS 174T cells with the O-linked glycosylation inhibitor benzyl- $\alpha$-GalNAc rendered the monolayer susceptible to amoebic attack, with results similar to those observed with the Chinese hamster ovary cell monolayer [33]. These studies highlight a critical role for Gal and GalNAc residues of colonic mucin in binding to and inhibiting the parasite Gal-lectin activity.

While E. histolytica may bind mucins with a high affinity, which is necessary for colonization, it can also degrade mucins to facilitate entry into the underlying epithelium, which causes disease. We have shown that cysteine proteases secreted from parasites participate in the disruption of the mucin polymer network, and consequently help to overcome the protective mucus barrier [34]. Parasite proteinases lead to proteolytic degradation of the terminal cysteine-rich D domains of the MUC2 polymer. Incubation with cysteine protease inhibitor E64 prevented the degradation of mucin [34]. As the $\mathrm{N}$ - and C-terminal cysteine-rich domains of mucin are involved in polymerization, we predicted they were the most likely targets of proteases. Furthermore, by treating recombinant cysteine-rich domains of MUC2 with proteases from E. histolytica trophozoites, we were able to show that the $\mathrm{C}$-terminal domain was specifically targeted at 2 sites by cysteine proteases, while the $\mathrm{N}$-terminal domain was resistant to proteolysis. We therefore concluded that the ma- jor cleavage site is predicted to depolymerize the MUC2 polymers, thereby disrupting the protective mucus gel [15]. More recently, we identified the specific cysteine protease 5 (EhCP5) of E. histolytica, as being responsible for colonic mucin degradation. This was further substantiated by the observation that antisense inhibition of EhCP5 in virulent $E$. histolytica trophozoites prevented the organism crossing the mucus barrier and disrupting mucin production in LS 174T and HT29 colonic cells [35]. Interestingly, EhCP5 is not present in the nonpathogenic Entamoeba dispar, suggesting a major role for this protease in the pathogenesis of intestinal amoebiasis.

Finally, when amoebas disrupt the mucus layer, the host cells exhibit an innate immune response that is characterized by the activation of NF- $\mathrm{B}$, recruitment of neutrophils and macrophages to the invasion site and production of several inflammatory cytokines, including IL$1 \beta$, IL- 6 and IL- 8 by epithelial cells $[36,37]$. Meanwhile, the parasite initiates a series of events that involve host calcium transport, caspase activation and destruction of host cells by apoptosis. E. histolytica initiates this apoptosis by directly activating the host cell's distal apoptotic machinery, since the normal apoptotic pathway mediators such as Fas/Fas ligands and TNF- $\alpha$ have no role in amoeba-induced apoptosis $[36,37]$. Since apoptosis, unlike necrosis, fails to induce an inflammatory response, the use of apoptotic destruction of host cells could serve to reduce the inflammatory response and provide an advantage for E. histolytica survival within the host. These systematic studies have unraveled mechanistically how a colonic pathogen can overcome luminal and innate epithelial barrier functions to cause disease.

\section{Regulation of Mucins by Bacterial Products}

Inducers of Mucin Production. Bacterial products such as lipopolysaccharides (LPS) and flagellin A from Gramnegative bacteria and lipoteichoic acids (LTA) from Gram-positive bacteria are the most common modulators of mucin production. They affect mainly MUC2 and MUC5AC.

LPS are predominantly found on the surface of Gramnegative bacteria. They are anchored to the outer bacterial surface by a lipid moiety (lipid A). LPS are a very strong stimulators of both innate and adaptive immunity. In mammalian cells, the recognition of bacterial LPS/ lipid A is achieved through the combined action of LPSbinding protein (LBP), membrane-bound or soluble lipoprotein, CD14 and TLR4 [38]. In the Gram-negative bacterium Pseudomonas aeruginosa, both LPS and flagellin play a role in altering mucin production [39-42]. LPS 
Fig. 3. Modulation of mucin expression by components of Gram-negative and Grampositive bacteria. LTA binds to host surface through platelet-activating factor receptor while LPS from Gram-negative bacteria bind through LBP and Toll-like receptors. Both the pathways converge at Ras and lead to the activation of NF- $\mathrm{B}$ and mucin production. Bacterial flagellin signals through binding to glycolipid Asialo-GM1. This stimulates the release of ATP which finally causes an increase in the level of intracellular calcium and stimulates downstream pathways. Binding to Toll-like receptors may also activate a sequence of signals involving PKC, DUOX1, ROS, TACE and EGFR activation, leading to downstream signaling. LTA = lipoteichoic acid; LPS = lipopolysaccharides; PAFR = platelet-activating factor receptor; $\mathrm{LBP}=\mathrm{LPS}$-binding protein; $\mathrm{G}=\mathrm{G}$ protein; ADAM10 = a disintegrin and metalloproteinase domain 10; HB-EGF = heparinbinding EGF-like growth factor; MAPK = mitogen-activated protein kinase; ERK = extracellular regulated kinase; $\mathrm{MEK}=$ MAPK ERK kinase; $\mathrm{PKC}=$ protein kinase C; DUOX1 = dual oxidase 1 ; ROS = reactive oxygen species; $\mathrm{TACE}=$ tumor necrosis factor- $\alpha$-converting enzyme; EGFR = epidermal growth factor receptor; TGF- $\alpha=$ transforming growth factor- $\alpha$.

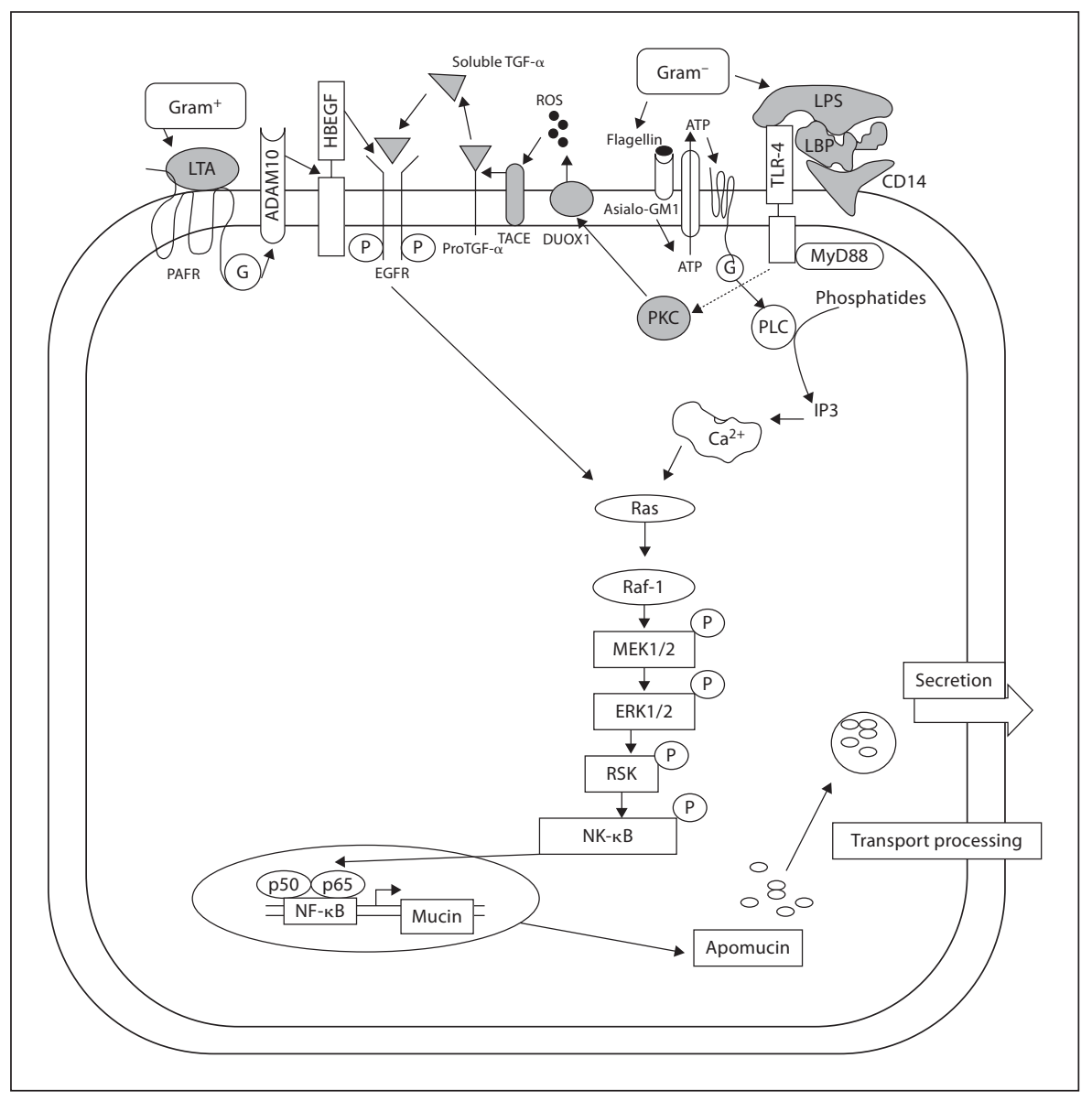

binds to LBP which in turn binds to CD14, and leads to activation of the Ras-MEK1/2-Erk1/2 pathway using the Toll-like receptor- 4 as a co-receptor [39]. Flagellin, on the other hand, binds to the surface glycolipid receptor, Asialo-GM1 [39, 40]. The activation of mucin transcription through Asialo-GM1 is calcium-dependent, as seen by an increase in calcium levels following the administration of flagellin or use of an agonist antibody in human HM3 cells [43]. Binding to Asialo-GM1 leads to the release of ATP and its subsequent binding to cell surface $G$ protein-coupled receptor (GPCR) [40, 43]. This activates phospholipase $\mathrm{C}$ and causes a subsequent increase in intracellular calcium levels. These events finally lead to the downstream activation of the Src-dependent Ras pathway $[40,43]$, leading to the activation of NF- $\mathrm{KB}$ and mucin transcription, as shown in figure 3.

Some aspects of mucin regulation have only been studied in airway epithelium and their counterparts in the intestinal epithelium need further investigation. For example, mucin gene expression in airway epithelium has been shown to involve the epidermal growth factor receptor (EGFR) [44]. These cell surface receptors can be activated in pathological conditions by LPS, by inflammatory stimuli such as TNF- $\alpha$, or by some types of oxidant damage that produce reactive oxygen species [40]. EGFR is a member of the receptor tyrosine kinase family [41], and binding of EGFR to its ligand leads to phosphorylation of its tyrosine. An external stimulus, such as a bacterial product or oxidant damage, activates enzyme dual oxidase- 1 . This leads to the production of a reactive oxygen species [40, $44]$ which activates the protease TNF- $\alpha$-converting enzyme that cleaves pro-TGF- $\alpha$ to release active TGF- $\alpha$. This in turn binds to and activates the EGFR, initiating MAPK signaling and mucin production [44].

Products of Gram-positive bacteria also affect mucin expression. The most common of these is LTA from Staphylococcus aureus and Staphylococcus pyrogenes and Listeriolysin $\mathrm{O}$ from Lysteria monocystogenes [45]. In the human epithelial cell line HM3, LTA binds and activates platelet-activating factor receptor, which is a cell surface 
G protein-coupled receptor [45]. This leads to activation of metalloproteinase ADAM10, which then cleaves the transmembrane heparin-binding EGF, and which in turn activates EGFR. This leads to the engagement of the Ras/ Raf/MEK/ERK/pp90 ${ }^{\text {rsk}} / \mathrm{NF}-\mathrm{kB}$ pathway and MUC2 transcription [45] (fig. 3).

Inhibitors of Mucin Production. In some cases, bacteria can also downregulate mucin synthesis and disrupt gastric mucosal integrity to enhance their survival. The best example of this is Helicobacter pylori, which colonizes the gastric mucosa. Infection with $H$. pylori leads to upregulation of the synthesis of inducible nitric oxide synthase (iNOS/NOS-2) and TNF- $\alpha$ production, along with enhancement of epithelial cell apoptosis. H. pylori infection causes a decrease in mucin synthesis, which leads to a loss of continuity in the mucus coat [46]. LPS from $H$. pylori lead to caspase-3 activation and apoptosis in a manner dependent on p38 MAPK (pro-apoptotic). Treatment of rat gastric mucosal segments with LPS from $H$. pylori has been shown to significantly inhibit mucin glycosylation and sulfation. These events may have a deleterious effect on mucin assembly and mucus coat continuity [47].

Similarly, LPS from the periodontopathic bacterium, Porphyromonas gingivalis, interfere with mucin synthesis and promote apoptosis in primary cultures of rat sublingual salivary-gland acinar cells [48]. LPS are capable of individual or simultaneous activation of p38, extracellular signal-regulated kinase (ERK) and the MAPK pathway. Inhibition of ERK increases the effect of LPS, while the blocking of p38 MAPK reduces the effect of LPS on repression of mucin synthesis. This illustrates the contrary responses to LPS, through the same pathway, in different cell types.

\section{Microbe-Derived Factors Alter the Chemical Composition of Mucin}

The defensive ability of mucin lies in its capacity to entrap microbes [27]. Adhesion to specific mucin epitopes is thought to facilitate mucus colonization by commensal bacteria, thereby providing a number of growth advantages. Accordingly, intestinal mucin is thought to dictate the composition of the bacterial community within the mucus layer. However, the mechanistic details of this selection process are poorly characterized. Most efforts have been focused on the identification of epithelial cell surface glycoprotein and glycolipid receptors for bacterial adhesins or toxins of enteric pathogens $[49,50]$. The mucus coat physically covers cell surface glycoconju- gates, and consequently it is thought to prevent adhesion of enteric pathogens. A number of studies with working models propose that soluble mucin epitopes bind to specific bacterial adhesins and prevent their attachment to similar epitopes on host cell surface receptors [27, 51]. It should be noted though, that since enteric pathogens are typically transient residents of the gut, such a defensive strategy would require constitutive host production of carbohydrate epitopes capable of binding to pathogen adhesins. The ecological issues associated with mucin's contrasting involvement in resistance to pathogens and facilitation of commensal bacteria are fascinating. They raise the question: what are the roles of the host, resident commensal bacteria and transient pathogens - or some combination of these - in the control of mucin composition? The answer probably lies in a direct interaction between intestinal microbes and epithelial cells.

\section{Other Goblet Cell Secretions in Innate Host Defense}

In addition to mucin production, goblet cells also produce 2 other important proteins: intestinal trefoil factor and resistin-like molecule- $\beta$ (RELM- $\beta$ ). Evidence suggests that these innate defense molecules may stabilize the mucin polymer and/or regulate mucin secretion. More importantly, these molecules may need a mucin medium to exert their biological functions. We consider them here based on these features.

\section{Intestinal Trefoil Factor}

Intestinal trefoil factor is a small cysteine-rich peptide belonging to the family of trefoil factors (TFF). In humans, 3 TFFs have been identified: TFF1, TFF2 and TFF3 (formerly intestinal trefoil) [52]. The expression patterns of these peptides vary in different anatomical portions of the gastrointestinal tract. TFF1 is a product of gastric surface mucus cells together with the MUC5AC. TFF2 is expressed in gastric mucus neck cells and cells at the pyloric glands together with MUC6. TFF3 is generated in intestinal goblet cells along with MUC2 [53].

TFFs are abundantly secreted onto the mucosal surface by goblet cells. All 3 TFFs are upregulated in surface epithelial cells at the margin of a gastric ulcer and also in IBD [53-55]. Secreted TFF acts on adjacent mucosal cell populations, either extracellularly to augment barrier function or intracellularly in transcriptional and signaling events. TFF response elements in TFF gene promoters 
also allow an increase in TFF expression through autoinduction and crossinduction of other TFFs, in addition to mucin expression and possibly tumor suppression. Trefoils seem to have 2 important roles: protection of epithelium and healing of injured mucosa. When mucosal injury occurs, trefoils are rapidly upregulated and stimulate repair by a process known as epithelial restitution [56]. They may also play a role in mucus stabilization, by interacting or crosslinking with mucins to aid in the formation of the gel layer [57]. Interestingly, TFF3 and mucin were together shown to be far more effective at protecting epithelial cells in vitro when compared with either one alone [57]. In addition, since trefoil peptides may be co-expressed with secreted mucins, there is a possible synergistic action in mucosal protection and repair between the two, since they are co-expressed in both normal and diseased mucosa. Mice deficient in TFF3 exhibit an increased sensitivity to intestinal damage. A study on TFF3 knockout mice demonstrated an impairment of colonic epithelial healing after oral dextran sulfate sodium (DSS) challenge, when compared with wild-type mice that developed a mild and transient colitis. Repletion of TFF3 leads to an improvement in the severe induced colitis in the knock-out mice [57].

\section{Resistin-Like Molecule- $\beta$}

RELM- $\beta$ (found in the inflammatory zone and also known as FIZZ2) is a member of resistin-like molecule (RELM) family. Other members in this family include resistin (FIZZ3), RELM- $\alpha$ (FIZZ1) and RELM- $\gamma$. Only resistin and RELM- $\beta$ are found in humans, whereas all 4 RELM family members have been identified in rodents. Members of the RELM family contain a cysteine-rich motif. Although no specific receptor for the RELMs has yet been reported, they are believed to act in both an autocrine and a paracrine fashion [58].

RELM- $\beta$ is expressed in the small and large intestine, within epithelial cells and, in particular, in goblet cells [59]. RELM- $\beta$ regulates colonic functions such as barrier integrity and inflammation susceptibility, as reported in the studies on RELM- $\beta$ knockout mice [60]. Disruption of the RELM- $\beta$ gene with the corresponding absence of local colonic RELM- $\beta$ proteins led to a reduction in the severity of colitis induced by either DSS or trinitrobenzene sulfonic acid $[60,61]$. RELM- $\beta$ expression is essentially undetectable in the colon of both germ-free immunocompetent and mice with severe combined immunodeficiency disease. However, RELM- $\beta$ can be induced within $48 \mathrm{~h}$ of transfer to a conventional environment, demonstrating that the expression of this gene is dependent upon bacterial colonization of the gut [59]. A recent study [58] has shown that MUC2 intrarectal injection of RELM- $\beta$ increases MUC2 secretion in the mouse colon. Furthermore, RELM- $\beta$ also upregulated MUC2 and M1/ MUC5AC expression in HT29-Cl.16E cell lines. These findings suggest that although both mucin and RELM- $\beta$ are secreted by goblet cells, RELM- $\beta$ can also act as an effective luminal mucin secretagogue.

\section{Mucin and Intestinal Disorders}

IBDs, which are characterized by chronic and relapsing gastrointestinal tract inflammation, are grouped into 2 broad categories: Crohn's disease and ulcerative colitis (UC). These are complex diseases that are caused by multiple environmental, immunological and genetic factors involving: (1) the immune system; (2) the microbes, and (3) the intestinal epithelial barrier. Evidence from animal models suggests that failure to suppress immune reactions to the abundant intestinal foreign antigen load is one of the critical factors in the manifestation of IBD. Maintaining the delicate balance of competence to respond to intestinal pathogens, without generating an inflammatory response to commensal organisms, appears to depend on a number of variables. These include the integrity of the mucosal and epithelial barriers, proinflammatory signaling pathways (especially via NF- $\kappa \mathrm{B}$ ) and regulation of innate and adaptive immune responses in the intestine.

Microbial factors are implicated in the initiation and/ or perpetuation of disease. Considering that the epithelium and its associated mucus layer form a physical barrier between bacteria and cells of the immune system, it is very likely that the epithelial barrier plays an important role in IBD. In support of this, there are several animal models in which a primary change in the integrity of the epithelium leads to IBD-like syndromes [9]. Mucins, as the primary constituents of extracellular mucus and the cellular barrier, are intimately associated with the etiological factors of IBD. A primary defect in mucin could breach the epithelial barrier or lead to altered mucosalbacterial interactions. On the other hand, the changing effects of immunological or bacterial factors during initial or ongoing inflammation could influence mucin production, thereby sustaining the chronic character of IBD. Previous studies have shown that mucin composition and its secretion are altered in IBD patients [9]. This alteration may be due to genetic mutations in mucin genes, changes 
in mucin mRNA or protein levels and changes in the extent of glycosylation, sulfation or the degradation of mucins [62]. Along with the changes in composition, recent studies [63] have demonstrated that structural changes in mucins also play a very important role in the genesis of IBDs. These authors concluded that mucin secreted in the intestine is prone to misfolding and/or aberrant assembly, which causes substantial stress on the endoplasmic reticulum, morphological goblet cell pathology and premature goblet cell apoptosis, which eventually leads to the development of chronic intestinal inflammation.

The mucus layer in UC is thinner than that found in normal controls [64] as the synthesis and secretion of MUC2 are reduced in the active stage of UC. This reduction in MUC2 secretion makes the colonic mucus barrier less protective. In addition, the function of MUC2 is altered. In active UC, MUC2 is undersulfated [65]. Alteration in sulfation of MUC2 may lead to a decreased protective capability of the mucin since the resistance to enzymatic degradation or bacterial stress is weakened. The depletion of goblet cells also causes the mucin layer to become thinner. Interestingly, anti-goblet cell auto-antibodies appear in $39 \%$ of patients with UC and may be responsible for the reduction in goblet cells [66]. In addition, the numbers of goblet cells are different in various portions of the colon. In UC patients, goblet cells are mainly reduced in the distal region, the main site of disease [67]. The colonic epithelium with reduced mucin is vulnerable to attack by chemicals or microorganisms and their products. Such attack would further injure the mucus layer. These abnormalities of mucin in UC can result in a loss of mucus barrier function and exposure of the mucosa to luminal agents that promote or perpetuate inflammation and induce anti-colon antibodies.

The physical state of the mucus, the change in the concentration of secreted mucin and the strong dependence of its physiochemical properties on environmental factors including ionic strength and $\mathrm{pH}$ play an important role in many diseases. In addition to serving as a barrier to bacteria, many bacteria reside within the mucus layer and possess adhesins that specifically bind to it [68]. These bacteria include pathogenic strains of Pseudomonas, Streptococcus and Pneumococcus. The alteration in mucin production is not only associated with IBDs but has been a hallmark of several pathological conditions. Some parasitic organisms are capable of producing their own layers of mucin to evade the immune system [69]. Overproduction of mucin is also involved in cystic fibrosis [70], bronchitis, asthma [71] and in middle-ear infections. Also, mucin underproduction is present in dry-eye syndromes [72] and in some forms of ulcer disease. Studies have also shown that mucin expression and composition is altered in cancers of epithelial origin [73].

In humans, it is extremely difficult to determine whether changes in bacterial colonization patterns in IBD are either a cause or a consequence of inflammation. To investigate this, normal enteric bacteria (NEB) were introduced in germ-free animals. It was found that germfree wild-type mice do not develop signs of inflammation after introduction of NEB [74, 75]. Moreover, germ-free mice produced normal amounts of MUC2 compared with mice harboring NEB, indicating that the presence of bacteria does not lead to altered mucus thickness [9]. Introduction of NEB into germ-free mice induced an increase in the sulfation of de novo synthesized MUC2. Normally, colonic mucins are highly sulfated and are generally considered to be more resistant to bacterial degradation. A colonic mucus layer composed of less-sulfated mucins is deemed less protective and this has been associated with IBD in humans $[65,76,77]$. Decreased levels of sulfation of MUC2 formed de novo suggest that NEB influences the structure and, possibly, the function of MUC2 during synthesis.

A number of recently developed rodent models of colitis are highly suitable for identifying the effects of the immune system on mucin expression. One of these animal models is the IL-10 $10^{-/-}$mouse, which spontaneously develops enterocolitis when maintained in conventional conditions and even in specified pathogen-free environments, but shows no evidence of colitis when kept in a germ-free environment [75]. In germ-free IL- $10^{-/-}$mice, at least a 10-fold decrease in MUC2 production was observed compared to germ-free wild-type mice [9]. Although the mucus layer was thinner, as a consequence of the decreased MUC2 synthesis, colitis was not manifested due to the absence of a bacterial-derived trigger from the lumen. On introduction of NEB, the IL- $10^{-/-}$mice rapidly developed symptoms of colitis, manifested by an infiltration of immune cells and induction of proinflammatory mediators such as IL-12. Goblet cell depletion, a characteristic feature of IBDs, was also observed [78]. The mechanism by which MUC2 synthesis is downregulated in IL-10 $0^{-/-}$mice remains unknown, yet these findings demonstrate that certain immune regulators directly or indirectly influence mucin production.

MUC2 $^{-/-}$mice are highly susceptible to IBD. Goblet cells in the absence of MUC2 lose their characteristic goblet-like shape, indicating that MUC2 is the major phenotypic determinant of goblet cells. $\mathrm{MUC}^{-/-}$mice are also extremely susceptible to cytotoxic luminal agents 
such as DSS. This was illustrated by the fact that treatment with DSS led to the occurrence of culminant colitis within days. Colitis induction was much more severe in $\mathrm{MUC2}^{-/-}$mice than in their wild-type counterparts [9], corroborating that MUC2 plays an essential role in epithelial protection. Another important cause of IBD is genetic variation. It has been shown that altered variable number tandem repeat regions in MUC3A are associated with UC, whereas single-nucleotide polymorphisms within the cytoplasmic C-terminus of the same gene predispose the carrier to Crohn's disease [79, 80]. A recently published meta-analysis of 11 genome-wide linkage studies for IBD revealed 38 significant IBD loci [81]. Moehle et al. [82] analyzed the chromosomal locations of all mucin genes and found that all mucin gene family members reside within or directly beside these IBD candidate loci. To comprehensively analyze whether mucin gene polymorphisms predispose for IBD, they performed allelic discrimination of mucin single-nucleotide polymorphisms in Crohn's disease, UC and control cohorts. They found that there was a statistically significant association of MUC2-V116M with Crohn's disease. They also identified different allelic frequencies for MUC4-A585S and MUC13-R502S in UC. These findings clearly suggest that genetic variations in the MUC4 and MUC13 genes may contribute to the development of IBD, although further large cohort studies with better defined control populations are required.

\section{Conclusions and Future Considerations}

Gastrointestinal mucus and its constituents play key roles in protecting the gut against enteric pathogens. Tremendous advancements have been made in the area of mucin gene discovery, cloning, structure and assembly. However, we still lack a basic understanding of mucin biology and the regulatory pathways involved in mucin biosynthesis and secretion. Studies unraveling the intricacies of the interaction between the mucosal barriers, immune mediators and the gut microbiota during different stages of disease pathogenesis - at both cellular and molecular levels - are eagerly awaited.

In this regard, primary attention should be given to the mechanisms by which gastrointestinal pathogens overcome the protective mucus barrier. Research is also needed to determine: (1) the putative mucin secretagogues released by pathogens; (2) the receptors and intracellular events involved in mucin exocytosis; (3) the intracellular mechanisms involved in accelerated secretion, and (4) the protein machinery involved in the release of stored and newly synthesized mucin.

It is also important that future research determines the level of the contribution made by mucosal barrier alterations to the pathogenesis of diseases like IBD, and the stage at which they become involved. In particular, researchers should try to cast light on whether mucosal barrier alterations play a primary role in pathogenesis or whether they play second fiddle to the extensive immune responses. This knowledge will help drug designers to focus on the upstream mechanisms. At present, the bulk of the research is focused on developing drugs that specifically block immune mediators, and there is little or no emphasis on producing medications to strengthen the mucosa.

Finally, a detailed insight into the role of mucin heterogeneity in different disease states and an understanding of genetic predisposition requires sustained attention. The 2 most abundant intestinal mucins, membranebound MUC3 and secretory MUC2, are clearly implicated in the pathogenesis of IBD. The linkage of IBD to different allelic variants of MUC3 gene has repeatedly been reported and confirmed, but the linkage to a genetic predisposition with the MUC2 gene still needs to be elucidated in a large and well-defined cohort of IBD patients and controls. The discovery of regulatory elements and identification of high and low producing single-nucleotide polymorphisms in the promoter region of the MUC2 and MUC3 genes will take research on the treatment of gastrointestinal diseases towards new horizons.

The most daunting task, however, is to translate the knowledge acquired from basic science and animal research into controlled clinical trials and, finally, to apply this to the treatment of patients. Basic science research needs to focus on further elucidation of the pathways, to generate more information on the targets that can strengthen mucosal barrier integrity. Clinical trials of potential future therapies such as probiotics and association studies analyzing genetic predisposition require a large patient cohort, well-characterized control populations and more collaborative multicenter trials.

\section{Acknowledgements}

Research in Dr. Chadee's laboratory is supported by grants from the Canadian Institute for Health Research, the Crohn's and Colitis Foundation of Canada, the Canadian Foundation for Innovation, the Natural Sciences and Engineering Research Council of Canada and the Canadian Association of Gastroenterology-Astra Zeneca-CIHR Research and Fellowship Awards. Dr. Chadee holds a Tier 1 Canada Research Chair in gastrointestinal inflammation. 


\section{References}

-1 McCracken VJ, Lorenz RG: The gastrointestinal ecosystem: a precarious alliance among epithelium, immunity and microbiota. Cell Microbiol 2001;3:1-11.

$\checkmark 2$ Gum JR Jr, Hicks JW, Gillespie AM, Carlson EJ, Komuves L, Karnik S, Hong JC, Epstein CJ, Kim YS: Goblet cell-specific expression mediated by the MUC2 mucin gene promoter in the intestine of transgenic mice. Am J Physiol 1999;276:G666-G676.

3 Moncada DM, Chadee K: Production, structure, and function of gastrointestinal mucins; in Blaser MJ (ed): Infections of the Gastrointestinal Tract. Philadelphia, Lippincott Williams \& Wilkins, 2002, pp 57-79.

$\checkmark 4$ Laboisse C, Jarry A, Branka JE, Merlin D, Bou-Hanna C, Vallette G: Recent aspects of the regulation of intestinal mucus secretion. Proc Nutr Soc 1996;55:259-264.

5 Forstner JF, Forstner GG: Gastrointestinal mucus; in Johnson LR (ed): Physiology of the Gastrointestinal Tract. New York, Raven Press, 1994, pp 1255-1283.

6 Habte HH, Mall AS, de Beer C, Lotz ZE, Kahn D: The role of crude human saliva and purified salivary MUC5B and MUC7 mucins in the inhibition of Human Immunodeficiency Virus type 1 in an inhibition assay. Virol J 2006;3:99.

$\checkmark 7$ Dekker J, Rossen JW, Buller HA, Einerhand AW: The MUC family: an obituary. Trends Biochem Sci 2002;27:126-131.

8 Perez-Vilar J, Hill RL: The structure and assembly of secreted mucins. J Biol Chem 1999; 274:31751-31754.

$>9$ Einerhand AW, Renes IB, Makkink MK, van der Sluis M, Buller HA, Dekker J: Role of mucins in inflammatory bowel disease: important lessons from experimental models. Eur J Gastroenterol Hepatol 2002;14:757-765.

10 Moncada DM, Kammanadiminti SJ, Chadee $\mathrm{K}$ : Mucin and Toll-like receptors in host defense against intestinal parasites. Trends Parasitol 2003;19:305-311.

$>11$ Vinall LE, Hill AS, Pigny P, Pratt WS, Toribara N, Gum JR, Kim YS, Porchet N, Aubert JP, Swallow DM: Variable number tandem repeat polymorphism of the mucin genes located in the complex on 11p15.5. Hum Genet 1998;102:357-366.

12 Herrmann A, Davies JR, Lindell G, Martensson S, Packer NH, Swallow DM, Carlstedt I: Studies on the 'insoluble' glycoprotein complex from human colon: identification of reduction-insensitive MUC2 oligomers and Cterminal cleavage. J Biol Chem 1999;274: 15828-15836.

13 Corfield AP, Wagner SA, Clamp JR, Kriaris MS, Hoskins LC: Mucin degradation in the human colon: production of sialidase, sialate $\mathrm{O}$-acetylesterase, $\mathrm{N}$-acetylneuraminate lyase, arylesterase, and glycosulfatase activities by strains of fecal bacteria. Infect Immun 1992;60:3971-3978.
14 Jentoft N: Why are proteins $O$-glycosylated? Trends Biochem Sci 1990;15:291-294.

15 Lidell ME, Moncada DM, Chadee K, Hansson GC: Entamoeba histolytica cysteine proteases cleave the MUC2 mucin in its C-terminal domain and dissolve the protective colonic mucus gel. Proc Natl Acad Sci USA 2006;103:9298-9303.

-16 Akiba Y, Guth PH, Engel E, Nastaskin I, Kaunitz JD: Dynamic regulation of mucus gel thickness in rat duodenum. Am J Physiol Gastrointest Liver Physiol 2000;279:G437G447.

17 Neutra M, Leblond CP: Synthesis of the carbohydrate of mucus in the golgi complex as shown by electron microscope radioautography of goblet cells from rats injected with glucose-H3. J Cell Biol 1966;30:119-136.

18 Forstner G: Signal transduction, packaging and secretion of mucins. Annu Rev Physiol 1995;57:585-605.

19 McCool DJ, Forstner JF, Forstner GG: Regulated and unregulated pathways for MUC2 mucin secretion in human colonic LS180 adenocarcinoma cells are distinct. Biochem J 1995;312:125-133.

20 Phillips TE, Phillips TH, Neutra MR: Regulation of intestinal goblet cell secretion. III. Isolated intestinal epithelium. Am J Physiol 1984;247:G674-G681.

21 Halm DR, Halm ST: Secretagogue response of goblet cells and columnar cells in human colonic crypts. Am J Physiol Cell Physiol 2000;278:C212-C233.

22 Forstner JF, Oliver MG, Sylvester FA: Production, structure and biologic relevance of gastrointestinal mucins; in Blaser MJ, Smith PD, Ravdin JI, Greenberg HB, Guerrant RL (eds): Infections of the Gastrointestinal Tract. New York, Raven Press, 1995, pp 71-88.

23 Villalobo A, Gabius H: Signaling pathways for transduction of the initial message of the glycocode into cellular responses. Acta Anat (Basel) 1998;161:110-129.

24 Belley A, Chadee K: Prostaglandin E(2) stimulates rat and human colonic mucin exocytosis via the $\mathrm{EP}(4)$ receptor. Gastroenterology $1999 ; 117: 1352-1362$.

25 Wright DH, Ford-Hutchinson AW, Chadee K, Metters KM: The human prostanoid DP receptor stimulates mucin secretion in LS174T cells. Br J Pharmacol 2000;131:1537-1545.

26 McCool DJ, Marcon MA, Forstner JF, Forstner GG: The T84 human colonic adenocarcinoma cell line produces mucin in culture and releases it in response to various secretagogues. Biochem J 1990;267:491-500.

27 Belley A, Keller K, Gottke M, Chadee K: Intestinal mucins in colonization and host defense against pathogens. Am J Trop Med Hyg 1999;60:10-15.

28 Kelly D, Conway S, Aminov R: Commensal gut bacteria: mechanisms of immune modulation. Trends Immunol 2005;26:326-333.
29 Lu L, Walker WA: Pathologic and physiologic interactions of bacteria with the gastrointestinal epithelium. Am J Clin Nutr 2001;73: 1124S-1130S.

30 Chadee K, Petri WA Jr, Innes DJ, Ravdin JI: Rat and human colonic mucins bind to and inhibit adherence lectin of Entamoeba histolytica. J Clin Invest 1987;80:1245-1254.

31 Petri WA Jr, Haque R, Mann BJ: The bittersweet interface of parasite and host: lectincarbohydrate interactions during human invasion by the parasite Entamoeba histolytica. Annu Rev Microbiol 2002;56:39-64.

32 Ravdin JI, Guerrant RL: Role of adherence in cytopathogenic mechanisms of Entamoeba histolytica: study with mammalian tissue culture cells and human erythrocytes. J Clin Invest 1981;68:1305-1313.

33 Belley A, Keller K, Grove J, Chadee K: Interaction of LS174T human colon cancer cell mucins with Entamoeba histolytica: an in vitro model for colonic disease. Gastroenterology 1996;111:1484-1492.

34 Moncada D, Keller K, Chadee K: Entamoeba histolytica cysteine proteinases disrupt the polymeric structure of colonic mucin and alter its protective function. Infect Immun 2003;71:838-844.

-35 Moncada D, Keller K, Ankri S, Mirelman D, Chadee K: Antisense inhibition of Entamoeba histolytica cysteine proteases inhibits colonic mucus degradation. Gastroenterology 2006;130:721-730.

-36 Seydel KB, Li E, Zhang Z, Stanley SL Jr: Epithelial cell-initiated inflammation plays a crucial role in early tissue damage in amebic infection of human intestine. Gastroenterology 1998;115:1446-1453.

37 Seydel KB, Stanley SL Jr: Entamoeba histolytica induces host cell death in amebic liver abscess by a non-Fas-dependent, non-tumor necrosis factor alpha-dependent pathway of apoptosis. Infect Immun 1998;66:29802983.

38 Alexander C, Rietschel ET: Bacterial lipopolysaccharides and innate immunity. J Endotoxin Res 2001;7:167-202.

-39 McNamara N, Basbaum C: Signaling networks controlling mucin production in response to Gram-positive and Gram-negative bacteria. Glycoconj J 2001;18:715-722.

40 Theodoropoulos G, Carraway KL: Molecular signaling in the regulation of mucins. J Cell Biochem 2007;102:1103-1116.

41 Andrianifahanana M, Moniaux N, Batra SK: Regulation of mucin expression: mechanistic aspects and implications for cancer and inflammatory diseases. Biochim Biophys Acta 2006;1765:189-222.

42 Nadel JA: Innate immune mucin production via epithelial cell surface signaling: relationship to allergic disease. Curr Opin Allergy Clin Immunol 2007;7:57-62. 
-43 McNamara N, Khong A, McKemy D, Caterina M, Boyer J, Julius D, Basbaum C: ATP transduces signals from ASGM1, a glycolipid that functions as a bacterial receptor. Proc Natl Acad Sci USA 2001;98:9086-9091.

44 Shao MX, Nadel JA: Dual oxidase 1-dependent MUC5AC mucin expression in cultured human airway epithelial cells. Proc Natl Acad Sci USA 2005;102:767-772.

-45 Lemjabbar H, Basbaum C: Platelet-activating factor receptor and ADAM10 mediate responses to Staphylococcus aureus in epithelial cells. Nat Med 2002;8:41-46.

-46 Slomiany BL, Slomiany A: Disruption in gastric mucin synthesis by Helicobacter pylori lipopolysaccharide involves ERK and p38 mitogen-activated protein kinase participation. Biochem Biophys Res Commun 2002; 294:220-224.

47 Liau YH, Lopez RA, Slomiany A, Slomiany BL: Helicobacter pylori lipopolysaccharide effect on the synthesis and secretion of gastric sulfomucin. Biochem Biophys Res Commun 1992;184:1411-1417.

-48 Slomiany BL, Slomiany A: Porphyromonas gingivalis lipopolysaccharide interferes with salivary mucin synthesis through inducible nitric oxide synthase activation by ERK and p38 kinase. Biochem Biophys Res Commun 2002;297:1149-1153.

-49 Chu SH, Walker WA: Bacterial toxin interaction with the developing intestine. Gastroenterology 1993;104:916-925.

50 Shah U, Walker WA: Adverse host responses to bacterial toxins in human infants. J Nutr 2000;130:420S-425S.

51 Gibbons RJ: Review and discussion of role of mucus in mucosal defense; in Strober W, Hanson LA, Sell KW (eds): Recent Advances In Mucosal Immunity. New York, Raven Press, 1982, pp 343-521.

52 Suemori S, Lynch-Devaney K, Podolsky DK: Identification and characterization of rat intestinal trefoil factor: tissue- and cell-specific member of the trefoil protein family. Proc Natl Acad Sci USA 1991;88:11017-11021.

53 Podolsky DK: Mucosal immunity and inflammation. V. Innate mechanisms of mucosal defense and repair: the best offense is a good defense. Am J Physiol 1999;277:G495G499.

-54 Hauser F, Poulsom R, Chinery R, Rogers LA, Hanby AM, Wright NA, Hoffmann W: hP1. $\mathrm{B}$, a human $\mathrm{P}$-domain peptide homologous with rat intestinal trefoil factor, is expressed also in the ulcer-associated cell lineage and the uterus. Proc Natl Acad Sci USA 1993;90: 6961-6965.

55 Poulsom R, Begos DE, Modlin IM: Molecular aspects of restitution: functions of trefoil peptides. Yale J Biol Med 1996;69:137-146.

56 Sasaki M, Ikeda H, Ohira S, Ishikawa A, Nakanuma Y: Expression of trefoil factor family 1,2 , and 3 peptide is augmented in hepatolithiasis. Peptides 2004;25:763-770.
Kindon H, Pothoulakis C, Thim L, LynchDevaney K, Podolsky DK: Trefoil peptide protection of intestinal epithelial barrier function: cooperative interaction with mucin glycoprotein. Gastroenterology 1995; 109:516-523.

58 Krimi RB, Kotelevets L, Dubuquoy L, Plaisancie P, Walker F, Lehy T, Desreumaux P, Van Seuningen I, Chastre E, Forgue-Lafitte ME, Marie JC: Resistin-like molecule beta regulates intestinal mucous secretion and curtails TNBS-induced colitis in mice. Inflamm Bowel Dis 2008;14:931-941.

59 He W, Wang ML, Jiang HQ, Steppan CM, Shin ME, Thurnheer MC, Cebra JJ, Lazar MA, Wu GD: Bacterial colonization leads to the colonic secretion of RELMbeta/FIZZ2, a novel goblet cell-specific protein. Gastroenterology 2003;125:1388-1397.

60 Hogan SP, Seidu L, Blanchard C, Groschwitz K, Mishra A, Karow ML, Ahrens R, Artis D, Murphy AJ, Valenzuela DM, Yancopoulos GD, Rothenberg ME: Resistin-like molecule beta regulates innate colonic function: bar rier integrity and inflammation susceptibility. J Allergy Clin Immunol 2006;118:257268.

61 McVay LD, Keilbaugh SA, Wong TM, Kierstein S, Shin ME, Lehrke M, Lefterova MI, Shifflett DE, Barnes SL, Cominelli F, Cohn SM, Hecht G, Lazar MA, Haczku A, Wu GD: Absence of bacterially induced RELM- $\beta$ reduces injury in the dextran sodium sulfate model of colitis. J Clin Invest 2006;116:29142923.

62 Morita H, Kettlewell MG, Jewell DP, Kent PW: Glycosylation and sulphation of colonic mucus glycoproteins in patients with ulcerative colitis and in healthy subjects. Gut 1993;34:926-932.

63 Shaoul R, Karban A, Weiss B, Reif S, Wasserman D, Pacht A, Eliakim R, Wardi J, Shirin $\mathrm{H}$, Wine E, Leshinsky-Silver E, Levine A: NOD2/CARD15 mutations and presence of granulomas in pediatric and adult Crohn's disease. Inflamm Bowel Dis 2004;10:709714.

64 Pullan RD, Thomas GA, Rhodes M, Newcombe RG, Williams GT, Allen A, Rhodes J: Thickness of adherent mucus gel on colonic mucosa in humans and its relevance to colitis. Gut 1994;35:353-359.

65 Van Klinken BJ, Van der Wal JW, Einerhand AW, Buller HA, Dekker J: Sulphation and secretion of the predominant secretory human colonic mucin MUC2 in ulcerative colitis. Gut 1999;44:387-393.

66 Folwaczny C, Noehl N, Tschop K, Endres SP, Heldwein W, Loeschke K, Fricke H: Goblet cell autoantibodies in patients with inflammatory bowel disease and their first-degree relatives. Gastroenterology 1997;113:101106.

67 Smithson JE, Campbell A, Andrews JM, Milton JD, Pigott R, Jewell DP: Altered expression of mucins throughout the colon in ulcerative colitis. Gut 1997;40:234-240.
68 Scharfman A, Lamblin G, Roussel P: Interactions between human respiratory mucins and pathogens. Biochem Soc Trans 1995;23: 836-839.

69 Jain M, Karan D, Batra SK, Varshney GC: Mucins in protozoan parasites. Front Biosci 2001;6:D1276-D1283.

70 Boucher RC: New concepts of the pathogenesis of cystic fibrosis lung disease. Eur Respir J 2004;23:146-158.

71 Rose MC: Mucins: structure, function, and role in pulmonary diseases. Am J Physiol 1992;263:L413-L429.

72 Argueso P, Gipson IK: Epithelial mucins of the ocular surface: structure, biosynthesis and function. Exp Eye Res 2001;73:281-289.

73 Hollingsworth MA, Swanson BJ: Mucins in cancer: protection and control of the cell surface. Nat Rev Cancer 2004;4:45-60.

74 Sartor RB: The influence of normal microbial flora on the development of chronic mucosal inflammation. Res Immunol 1997;148: 567-576.

75 Sellon RK, Tonkonogy S, Schultz M, Dieleman LA, Grenther W, Balish E, Rennick DM Sartor RB: Resident enteric bacteria are necessary for development of spontaneous colitis and immune system activation in interleukin-10-deficient mice. Infect Immun 1998;66:5224-5231.

76 Tytgat KM, Buller HA, Opdam FJ, Kim YS, Einerhand AW, Dekker J: Biosynthesis of human colonic mucin: Muc2 is the prominent secretory mucin. Gastroenterology 1994; 107:1352-1363

77 Tsai HH, Dwarakanath AD, Hart CA, Milton JD, Rhodes JM: Increased faecal mucin sulphatase activity in ulcerative colitis: a potential target for treatment. Gut 1995;36: 570-576.

78 McCormick DA, Horton LW, Mee AS: Mucin depletion in inflammatory bowel disease. J Clin Pathol 1990;43:143-146.

79 Kyo K, Muto T, Nagawa H, Lathrop GM, Nakamura Y: Associations of distinct variants of the intestinal mucin gene MUC3A with ulcerative colitis and Crohn's disease. J Hum Genet 2001;46:5-20.

80 Kyo K, Parkes M, Takei Y, Nishimori H, Vyas P, Satsangi J, Simmons J, Nagawa H, Baba S, Jewell D, Muto T, Lathrop GM, Nakamura Y: Association of ulcerative colitis with rare VNTR alleles of the human intestinal mucin gene, MUC3. Hum Mol Genet 1999;8:307311.

81 Brant SR, Shugart YY: Inflammatory bowel disease gene hunting by linkage analysis: rationale, methodology, and present status of the field. Inflamm Bowel Dis 2004;10:300311.

82 Moehle C, Ackermann N, Langmann T, Aslanidis C, Kel A, Kel-Margoulis O, Schmitz-Madry A, Zahn A, Stremmel W, Schmitz G: Aberrant intestinal expression and allelic variants of mucin genes associated with inflammatory bowel disease. J Mol Med 2006;84:1055-1066. 